\title{
La heurística LDMTP: Una metodología híbrida basada en el problema de transporte para el diseño óptimo de la distribución de planta
}

\author{
The LDMTP Heuristic: A Hybrid Methodology Based on the Transportation \\ Problem for the Optimal Design of Plant Layout
}

\author{
González-Longoria Héctor Manuel \\ Universidad Tecnológica Santa Catarina \\ Departamento de Ingeniería en Sistemas Productivos \\ Correo:hmgonzalez@utsc.edu.mx
}

Información del artículo: recibido: junio de 2015, reevaluado: septiembre de 2015 y enero de 2016, aceptado: junio de 2016

\section{Resumen}

En este trabajo se presenta una heurística eficiente e innovadora para determinar el diseño óptimo de la distribución de planta (layout) que minimiza el costo total de flujo de materiales dentro de una empresa, incluyendo reglas para realizar la distribución inicial y el proceso lógico para reducir el trabajo de detectar el arreglo que ofrece las mejores oportunidades para reducir el costo total de la distribución. Esta nueva heurística se basa en el uso modificado del problema de transporte clásico (PTC), que es una herramienta propia de la programación lineal y que forma parte de la investigación de operaciones. La heurística LDMTP de creación propia ha demostrado que es eficiente para resolver el diseño óptimo de la distribución de planta, debido a que este es un método selectivo capaz de distinguir el mejor orden de asignación de todos los departamentos que conforman el layout, incluyendo criterios de forma (largo y ancho) para cada departamento asignado. El proceso de establecer la asignación inicial de la heurística LDMTP no es un asunto subjetivo, debido a que la elección del mejor orden de asignación de los departamentos que conforman la distribución inicial sigue una serie de pasos y tiene hasta 4 diferentes criterios de desempate, lo cual favorece que se elija el arreglo de departamentos que puede aportar la mayor contribución a la reducción del costo total de la distribución.

\section{Descriptores:}

- distribución de planta

- problema de transporte modificado

- programación lineal

- heurística

- CRAFT

- prueba de Anderson-Darling

- prueba paramétrica de los rangos con signo 


\begin{abstract}
This paper presents an efficient heuristics to determine the optimal design of plant layout, that minimizes the total cost of material flow within a company, including rules for the initial distribution and logical process to reduce the work to detect the arrangement that offers the best opportunities to reduce total cost process distribution. This new heuristic apply the modified use of Classic Transportation Problem (CTP), which is a proprietary tool of Linear Programming and part of the Operations Research. The LDMTP heuristic of own creation has proven to be efficient for solving the optimal design of plant layout due to is a selective method that is capable of distinguishing the best assignment order of all departments that make up the layout including criteria form (long and width) for each assigned department. Establishing the initial allocation of LDMTP heuristics is not a subjective matter because choosing the best assignment order of departments that make up the initial distribution follows a series of steps and has up to 4 different tiebreakers, so which favors the settlement of departments can make the greatest contribution is chosen to reducing the total cost of distribution.
\end{abstract}

\section{Keywords:}

- plant layout

- modified transportation problem

- linear programming

- heuristics, CRAFT

- Anderson -Darling test

- parametric test of ranges with sign

\section{Introducción}

El diseño óptimo de la distribución de planta Optimal Design of Plant Layout (ODPL) es un problema ampliamente estudiado que repercute en el incremento de la productividad y disminución de los costos de fabricación de una empresa. En este artículo se muestra una heurística de creación propia, que es eficiente e innovadora, ya que está basada en el uso del problema de transporte modificado (PTM) de la programación lineal, la cual se denomina: Diseño de la distribución de planta basada en el problema de transporte modificado, Layout Design based on the Modified Transportation Problem (LDMTP).

Las aplicaciones usuales de la programación lineal (PL) son el método simplex (MS), el problema de transporte (PT) y el método de asignación (MA). Mejía (2012) sugiere el uso de algoritmos genéticos para el problema de OPDL, lo cual involucra el uso de lenguajes de progra- mación. No existe ninguna aplicación encontrada hasta ahora del diseño óptimo de la distribución de planta (ODPL) basada en el problema de transporte que se encuentre referenciada en la literatura. Esta es la razón de la innovación que aporta esta heurística.

Para entender cómo se construye una tabla del problema de transporte se anexa como ejemplo un extracto de la tabla 8.22 de Hillier \& Lieberman en la figura 1.

$$
\$=\frac{\$ 15}{\text { pieza }} \quad 10 \text { piezas }=\$ 150
$$

La formulación de LDMTP como un problema de transporte se muestra en la figura 2, que guarda una analogía con la figura 1.

$$
\$=\frac{\$ 6}{\mathrm{~m}} \quad 25 \mathrm{mts}=\$ 150
$$

1 TABLA 8.22 Parte de la segunda tabla símplex de transporte que muestra los cambios en la solución BF

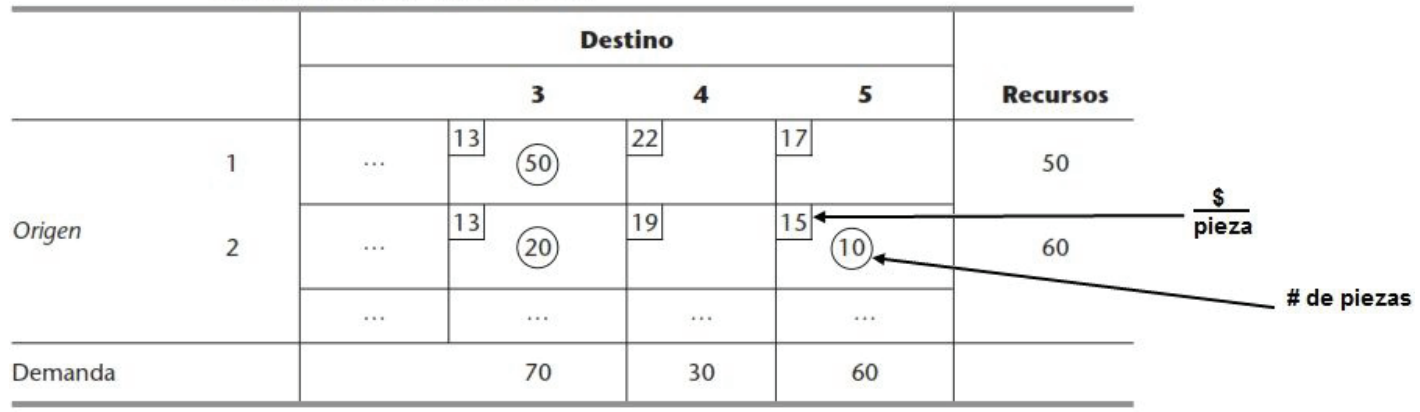

Figura 1. Extracto de tabla $8.22 \mathrm{H} \& \mathrm{~L}$ del problema de transporte

Fuente: Hillier \& Lieberman (2001) 


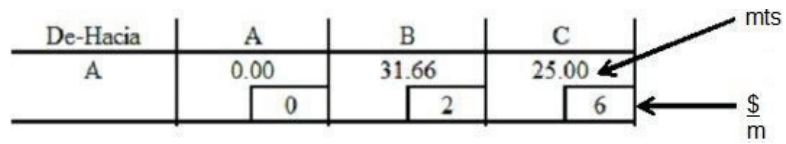

Figura 2. Interpretación gráfica del método LDMTP Fuente: elaboración propia (2016)

La heurística LDMTP se usa para el diseño de layout, emplea criterios de asignación como las rutinas de construcción (CORELAP, ALDEP). La optimización de la heurística se basa en minimizar el costo de manejo de materiales como las rutinas de mejoramiento (CRAFT, COFAD, PLANET, MCRAFT, etcétera). Como combina las características de los dos tipos de rutinas: de construcción y de mejoramiento, esta heurística se puede considerar híbrida, la cual no se ha detectado en la literatura.

\section{Desarrollo}

La formulación matemática del diseño óptimo de la distribución de planta que es el objetivo de esta heurística, se describe por Wang et al. (2005) y John et al. (2013) como:

$\min M F F C=\sum \sum C_{i j} f_{i j} d_{i j}$

donde

MFFC $=$ Costo del factor de flujo de material

$\mathrm{C}_{\mathrm{ij}} \quad$ = Costo de transporte de material unitario entre los departamentos i y j

$\mathrm{f}_{\mathrm{ij}} \quad=$ Flujo de material entre los departamentos i $\mathrm{y}$ j

$\mathrm{d}_{\mathrm{ij}} \quad=$ Distancia rectilínea entre los departamentos $\mathrm{i}$ y j

$\mathrm{F}_{\mathrm{ij}}=\mathrm{C}_{\mathrm{ij}} \mathrm{f}_{\mathrm{ij}}=$ Producto costo-flujo de transporte de material unitario entre los departamentos i y j

La aplicación de la ecuación 1 a condiciones especiales se muestra en las ecuaciones 2, 3 y 4 .

Si i $=j$, entonces $C_{i j}=0, f_{i j}=0, d_{i j}=0, y \min M F F C=0(2)$

Si i $\neq \mathrm{j} \mathrm{y}_{\mathrm{ij}}=0$, entonces $\min \mathrm{MFFC}=0$

Si $\mathrm{i} \neq \mathrm{j} \mathrm{y}_{\mathrm{ij}}>0$, entonces $C_{\mathrm{ij}}>0, \mathrm{~d}_{\mathrm{ij}}>0, \mathrm{y} \min \mathrm{MFFC}>0(4)$

\section{Criterio de forma de departamento (ancho y largo)}

En este método la relación de forma $\left(\operatorname{Largo} \mathrm{L}_{\mathrm{i}} /\right.$ Ancho $\left._{\mathrm{i}}\right)$ usualmente se ajusta para cumplir con la condición siguiente $\frac{\text { Largo } L_{i}}{\text { Ancho } W_{i}} \geq \frac{L_{T}}{W_{T}}$

donde

$\mathrm{L}_{\mathrm{i}} \quad$ = Largo del departamento i y $\mathrm{W}_{\mathrm{i}}=$ Ancho del departamento i

$\mathrm{L}_{\mathrm{T}}=$ Largo total del layout y $\mathrm{W}_{\mathrm{T}}=$ Ancho total del layout

Se usan los valores de $\mathrm{L}_{\mathrm{i}}$ y $\mathrm{W}_{\mathrm{i}}$ obtenidos para calcular un factor de forma sugerido por Bozer et al. (2005) con la fórmula siguiente

$\Omega_{\mathrm{i}}=\frac{\mathrm{P}_{\mathrm{i}}}{4 \sqrt{\mathrm{A}_{\mathrm{i}}}}$

donde se sugiere $\Omega_{\mathrm{i}} \leq 1.5$

$\mathrm{P}_{\mathrm{i}}=$ perímetro del departamento $\mathrm{i}$

$\mathrm{A}_{\mathrm{i}}=$ Área del departamento $\mathrm{i}$

Para una figura rectangular o cuadrada, la ecuación 6 se puede expresar de la siguiente forma

$\Omega_{\mathrm{i}}=\frac{2\left(\mathrm{~L}_{\mathrm{i}}+\mathrm{W}_{\mathrm{i}}\right)}{4 \sqrt{\left(\mathrm{L}_{\mathrm{i}} \times \mathrm{W}_{\mathrm{i}}\right)}}$

En caso de que exista un conflicto con la condición de forma y se cumpla la condición de la ecuación 5 , pero se rebase el valor de $\Omega_{\mathrm{i}}$ de la ecuación 6, predomina la condición de la ecuación 5 .

\section{Pasos de la aplicación de la heurística}

Los pasos y criterios de asignación y ubicación para los departamentos que integran el layout son los siguientes:

Paso 1. Determinar $\mathrm{F}_{\mathrm{ij}}^{+}$

Paso 2. Determinar $D_{1}=$ Departamento con mayor área de $\mathrm{F}_{\mathrm{ij}}^{+}$. ¿Se puede definir $\mathrm{D}_{1}$ ?

Sí. Ir al paso $3 \quad$ No. Ir al paso 2.1

Paso 2.1. En caso de no poder determinar claramente $D_{1}$ debido a que los dos departamentos que integran $\mathrm{F}_{\mathrm{ij}}^{+}$ tengan la misma área, usar los criterios siguientes para tratar de desempatar. 
$\sum \mathrm{F}_{\mathrm{D} 1}{ }^{+}=\operatorname{Max}\left(\sum \mathrm{F}_{\mathrm{D} 1, \mathrm{r}}, \sum \mathrm{F}_{\mathrm{D} 1 \mathrm{j}}\right) \sum \mathrm{F}_{\mathrm{D} 2}{ }^{+}=\operatorname{Max}\left(\sum \mathrm{F}_{\mathrm{D} 2, \mathrm{r}}, \sum \mathrm{F}_{\mathrm{D} 2, \mathrm{j}}\right) \mathrm{F}^{+}=$ $\operatorname{Max}\left(\sum \mathrm{F}_{\mathrm{D} 1}{ }^{+}, \sum \mathrm{F}_{\mathrm{D} 2}{ }^{+}\right)$. ¿Se puede definir $\mathrm{D}_{1}$ ?

Sí. Ir al paso $3 \quad$ No. Ir al paso 2.2

Paso 2.2. En caso de no poder determinar claramente $D_{1}$ debido a que los dos departamentos que integran $\mathrm{F}_{\mathrm{ij}}{ }^{+}$ tengan la misma área, usar los criterios siguientes para tratar de desempatar.

$\sum \mathrm{F}_{\mathrm{D} 1}{ }^{-}=\operatorname{Min}\left(\sum \mathrm{F}_{\mathrm{D} 1, \mathrm{r}}, \sum \mathrm{F}_{\mathrm{D} 1, \mathrm{j}}\right) \mathrm{F}_{\mathrm{D} 2}{ }^{-}=\operatorname{Min}\left(\sum \mathrm{F}_{\mathrm{D} 2, \mathrm{r}} \sum \mathrm{F}_{\mathrm{D} 2, \mathrm{j}}\right) \quad \mathrm{F}^{-}=$ $\operatorname{Max}\left(\sum \mathrm{F}_{\mathrm{D} 1}, \sum \mathrm{F}_{\mathrm{D} 2}\right) \cdot$ ¿Se puede definir $\mathrm{D}_{1}$ ?

Sí. Ir al paso 3. $\quad$ No. Ir al paso 2.3

Paso 2.3. Asignación: Libre elección (romper empates arbitariamente). Ubicación: $d_{i j}{ }^{-}$(distancia mínima entre departamentos).

Paso 3. El departamento $D_{1}$ asignado debe cumplir con los lineamientos de forma sugeridos por las ecuaciones 5, 6 y 7. La longitud del departamento $D_{1}$ debe estar alineada a la longitud del área disponible, ya que esta alineación asegura que la distancia entre departamentos $\mathrm{d}_{\mathrm{ij}}$ sea mínima.

Paso 4. Ya asignado $D_{1}$, se asigna $D_{2}$ (Departamento no asignado en primer lugar de $\mathrm{F}_{\mathrm{ij}}{ }^{+}$) usando los mismos lineamientos de forma para alinear $D_{1}$.

Paso 5. Los criterios para asignación a partir del tercer departamento son: $(\mathrm{k} \geq 3)$

$\mathrm{F}_{\mathrm{k}}^{+}=\operatorname{Max}\left(\mathrm{F}_{\mathrm{ik}}, \mathrm{F}_{\mathrm{j} \mathrm{k}}\right) \quad \mathrm{D}_{\mathrm{k}}$ debe estar relacionado con $\mathrm{D}_{1}$ o $\mathrm{D}_{2}$ o hasta $\mathrm{D}_{\mathrm{k}-1}$.

Paso 6. Repetir el procedimiento a partir del paso 5 hasta realizar todas las asignaciones de departamentos tomando en cuenta los lineamientos de forma sugeridos por Bozer et al. (2005).

Paso 7. Realizar la distribución inicial de la Forma $1\left(\mathrm{D}_{1}\right.$, $\mathrm{D}_{2}, \mathrm{D}_{3}$ ). Determinar el costo total de la primera distribución de planta.

Paso 7.1. Si para el tercer departamento a asignar, se cumple que: $\mathrm{D}_{3}$ debe estar junto a $\mathrm{D}_{1}$ y $\mathrm{A}_{\mathrm{D} 3}=\mathrm{A}_{\mathrm{D} 2}=\mathrm{A}_{\mathrm{D} 1}$, se debe verificar la posibilidad de usar criterios alternativos para asignar el tercer departamento.

Sí. Ir al paso 7.2. $\quad$ No. Ir al paso 8

Paso 7.2. Reorganizar el layout de la forma siguiente:
$\mathrm{D}_{1 \text { modificado }}=\mathrm{D}_{2 \text { original }} \quad \mathrm{D}_{2 \text { modificado }}=\mathrm{D}_{1 \text { original }} \quad \mathrm{D}_{3 \text { modificado }}=$
$\mathrm{D}_{3 \text { original }}$

Realizar la distribución inicial de la Forma $2\left(\mathrm{D}_{2}, \mathrm{D}_{1}\right.$, $\mathrm{D}_{3}$ ). Determinar el costo de la distribución inicial de la Forma 2 y comparar con la Forma 1. ¿\$Forma2 < \$Forma1?

Sí. Ir al paso 7.3

No. Ir al paso 8 y hacer el análisis con la Forma 1

Paso 7.3. Hacer todo el análisis posterior con la Forma 2.

Se presenta un compendio de los casos que cumplen con la condición del paso 7.1. (Tabla 1).

$\mathrm{D}_{1}=$ Departamento $1 \quad \mathrm{D}_{2}=$ Departamento 2

$\mathrm{D}_{3}=$ Departamento 3

$73 \%$ de los casos (8 de 11) ofreció mejores resultados intercambiando los departamentos, usando $\mathrm{D}_{2}, \mathrm{D}_{1}, \mathrm{D}_{3}$ en vez de $\mathrm{D}_{1}, \mathrm{D}_{2}, \mathrm{D}_{3}$ lo cual generó una reducción de $15 \%(\$ 166,474-\$ 196,776 / \$ 196,776 \times 100)$ del valor inicial de la primera distribución.

Paso 8. Establecer todos los intercambios válidos entre dos departamentos (Nahmias, 1999) que pueden ser:

a) Que los dos departamentos a intercambiar tengan una frontera en común.

b) Que los dos departamentos a intercambiar tengan la misma área, aunque no sean contiguos.

Nota: El intercambio que se realizó para generar un nuevo layout, no es un intercambio válido para esa nueva distribución, pero sí para distribuciones siguientes. Por ejemplo, si el intercambio A-B es el origen del $2^{\circ}$ layout (no es un intercambio válido para este layout), pero sí puede considerarse un intercambio válido a partir del 3er layout (siempre y cuando se cumpla alguna de las condiciones mencionadas en el paso 8).

Paso 9. Realizar todos los intercambios válidos entre dos departamentos (intercambio de coordenadas: criterio sugerido por CRAFT). Determinar si existe un intercambio que pudiera reducir el costo total de la distribución de planta. ¿Existe un intercambio para el cual el costo del intercambio < costo del origen?

Sí. Ir al paso $10 \quad$ No. Esta es la distribución de planta (layout) óptima 
Tabla 1. Casos con reorganización del orden de asignación de los tres primeros departamentos. Fuente: elaboración propia (2016)

\begin{tabular}{|c|c|c|c|c|}
\hline \multirow{2}{*}{$\#$} & \multirow{2}{*}{ Caso } & \multirow{2}{*}{ Tamaño de la matriz } & \multicolumn{2}{|c|}{ Costo de la distribución inicial final } \\
\hline & & & $\mathrm{D}_{1}, \mathrm{D}_{2}, \mathrm{D}_{3}$ & $\mathrm{D}_{2}, \mathrm{D}_{1}, \mathrm{D}_{3}$ \\
\hline 1 & Ejercicio 3.2 resuelto Chary (1995) & $3 \times 3$ & $\$ 18$ & $\$ 15$ \\
\hline 2 & Problema 6 Chase (2001) & $4 \times 4$ & $\$ 22,875$ & $\$ 7,750$ \\
\hline 3 & Ejemplo Galindo y Tapia (2013) & $5 \times 5$ & $\$ 1,610$ & $\$ 1,320$ \\
\hline 4 & Problema 9.2 Heizer (2001) & $6 \times 6$ & $\$ 13,975$ & $\$ 11,625$ \\
\hline 5 & Ejercicio 9.1 Heizer (2001) resuelto & $8 \times 8$ & $\$ 5,350$ & $\$ 5,000$ \\
\hline 6 & Problema 3.1 Chary (1995) & $9 \times 9$ & $\$ 18,300$ & $\$ 17,400$ \\
\hline 7 & Ejemplo 3.1 Chary (1995) & $9 \times 9$ & $\$ 445$ & $\$ 475$ \\
\hline 8 & Ejemplo Tabla 2. Jithavech et al. (2010) & $9 \times 9$ & $\$ 132,640$ & $\$ 121,440$ \\
\hline 9 & Ejemplo Adi (2013) & $10 \times 10$ & $\$ 2,008$ & $\$ 1,924$ \\
\hline 10 & $\begin{array}{c}\text { Ejemplo Tabla } 11 \text { E2. Jithavech } \\
\text { et al. (2010) }\end{array}$ & $16 \times 16$ & $\$ 234,420$ & $\$ 284,780$ \\
\hline 11 & $\begin{array}{c}\text { Ejemplo Tabla } 16 \text { E7. Jithavech } \\
\text { et al. (2010) }\end{array}$ & $25 \times 25$ & $\$ 604,290$ & $\$ 822,540$ \\
\hline- & Suma $(1,2,3,4,5,6,8$ y 9$)$ & - & $\$ 196,776$ & $\$ 166,474$ \\
\hline
\end{tabular}

Paso 10. Modificar el layout, recalcular los centroides y determinar el costo del origen del nuevo layout.

Nota: Si un departamento no tiene una forma rectangular o cuadrada, es necesario aplicar las fórmulas para el cálculo de centroides (Nahmias, 1999) mostradas en las ecuaciones 8 y 9 .

$M_{x}=\frac{\sum_{i=1}^{i=k}\left[\left(X_{2 i}{ }^{2}-X_{1 i}{ }^{2}\right)\left(Y_{2 i}-Y_{1 i}\right)\right]}{2}$

$M_{y}=\frac{\sum_{i=1}^{i=k}\left[\left(Y_{2 i}{ }^{2}-Y_{1 i}{ }^{2}\right)\left(X_{2 i}-X_{1 i}\right)\right]}{2}$

$\overline{\mathrm{X}}=\frac{\mathrm{M}_{\mathrm{x}}}{\mathrm{A}} \overline{\mathrm{Y}}=\frac{\mathrm{M}_{\mathrm{y}}}{\mathrm{A}}$

$\mathrm{Mx}=$ Momento en " $\mathrm{X}$ " My $=$ Momento en " $Y$ "

$\bar{X}=$ Centroide en " $X " \quad \bar{Y}=$ Centroide en " $Y$ "

Paso 11. Repetir los pasos 8 al 10 (de ser necesario).

\section{Ejemplo de aplicación de la Heurística}

P1. Conflicto de criterios e intercambio de orden D2, D1, D3 (Problema 6-Chase et al., 2001)

Los datos de P1Forma 1 de la distribución inicial del método LDMTP se muestran en la tabla 2.

$F_{i j}=$ Producto costo-flujo de transporte de material unitario entre los departamentos i y $\mathrm{j}$ (valores en cuadro) $\sum \mathrm{F}_{\mathrm{ij}}=$ Sumatoria de $\mathrm{F}_{\mathrm{ij}}$ por fila o columna

\section{Pasos de la aplicación de la heurística}

Los pasos y criterios de asignación y ubicación para los departamentos que integran el layout son los siguientes:

Paso 1. Determinar $\mathrm{F}_{\mathrm{ij}}^{+}$

En nuestro caso, P1 Forma 1, $\mathrm{F}_{\mathrm{ij}}^{+}=110$ (intercambio A-D).

Paso 2. Determinar $\mathrm{D}_{1}=$ Departamento con mayor área de $\mathrm{F}_{\mathrm{ij}}^{+}$. ¿Se puede definir $\mathrm{D}_{1}$ ? 
Tabla 2. Datos de la distribución inicial del método LDMTP Forma 1 (Problema 6-Chase et al., 2001). Fuente: elaboración propia (2016)

\begin{tabular}{|c|c|c|c|c|c|}
\hline De-Hacia & $\mathbf{A}$ & B & $\mathrm{C}$ & D & $\sum \mathrm{F}_{\mathrm{ij}}$ \\
\hline $\mathbf{A}$ & 0 & 20 & 50 & 110 & 180 \\
\hline B & 0 & 0 & 20 & 10 & 30 \\
\hline C & 0 & 0 & 0 & 30 & 30 \\
\hline D & $\overline{0}$ & 0 & 0 & 0 & 0 \\
\hline$\sum F_{i j}$ & 0 & 20 & 70 & 150 & \\
\hline
\end{tabular}

En nuestro caso, P1 Forma 1, determinar D1 = Departamento con mayor área de $\mathrm{F}_{\mathrm{ij}}^{+}$(A y D 5000). En nuestro caso no se puede definir $\mathrm{D}_{1}$.

Paso 2.1. En caso de no poder determinar claramente $D_{1}$ debido a que los dos departamentos que integran $\mathrm{F}_{\mathrm{ij}}{ }^{+}$ tengan la misma área, usar los criterios siguientes para tratar de desempatar.

$\sum \mathrm{F}_{\mathrm{D} 1}{ }^{+}=\operatorname{Max}\left(\sum \mathrm{F}_{\mathrm{D} 1, \mathrm{i}^{\prime}}, \sum \mathrm{F}_{\mathrm{D} 1, \mathrm{j}}\right) \sum \mathrm{F}_{\mathrm{D} 2}{ }^{+}=\operatorname{Max}\left(\sum \mathrm{F}_{\mathrm{D} 2, \mathrm{i}^{\prime}} \sum \mathrm{F}_{\mathrm{D} 2, \mathrm{j}}\right) \mathrm{F}^{+}=$ $\operatorname{Max}\left(\sum \mathrm{F}_{\mathrm{D} 1}^{+}, \sum \mathrm{F}_{\mathrm{D} 2}{ }^{+}\right) \cdot i$ Se puede definir $\mathrm{D}_{1}$ ?

Sí. Ir al paso $3 \quad$ No. Ir al paso 2.2

$\sum \mathrm{F}_{\mathrm{D} 1, \mathrm{i}}=$ Suma de datos de $\mathrm{F}_{\mathrm{ij}}$ (Producto costo-flujo) del departamento $\mathrm{D}_{1}$ por fila

$\sum \mathrm{F}_{\mathrm{D} 1, \mathrm{j}}=$ Suma de datos de $\mathrm{F}_{\mathrm{ij}}$ (Producto costo flujo) del departamento $\mathrm{D}_{1}$ por columna

$\sum \mathrm{F}_{\mathrm{D} 2, \mathrm{i}}=$ Suma de datos de $\mathrm{F}_{\mathrm{ij}}$ (Producto costo-flujo) del departamento $\mathrm{D}_{2}$ por fila

$\sum \mathrm{F}_{\mathrm{D} 2, \mathrm{j}}=$ Suma de datos de $\mathrm{F}_{\mathrm{ij}}$ (Producto costo-flujo) del departamento $\mathrm{D}_{2}$ por columna

$\sum \mathrm{F}_{\mathrm{D} 1}{ }^{+}=$Valor máximo de $\left(\sum \mathrm{F}_{\mathrm{D} 1, \mathrm{i}}, \sum \mathrm{F}_{\mathrm{D} 1, \mathrm{j}}\right)$

$\sum \mathrm{F}_{\mathrm{D} 2}{ }^{+}=$Valor máximo de $\left(\sum \mathrm{F}_{\mathrm{D} 2, \mathrm{i}}, \sum \mathrm{F}_{\mathrm{D} 2, \mathrm{j}}\right)$

En nuestro caso P1 Forma 1, $\sum \mathrm{F}_{\mathrm{A}}+=\operatorname{Max}\left(\sum_{\mathrm{FA}, \mathrm{i}}, \sum_{\mathrm{FA}, \mathrm{j}}\right)=$ $\operatorname{Max}(180,0), \sum \mathrm{F}_{\mathrm{D}}^{+}=\operatorname{Max}\left(\sum \mathrm{F}_{\mathrm{D}, \mathrm{i}}, \sum \mathrm{F}_{\mathrm{D}, \mathrm{j}}\right)=\operatorname{Max}(150,150)$, $\mathrm{F}^{+}=\operatorname{Max}(180,150)=180$. Se define $\mathrm{D}_{1}=$ departamento A.

Nota: $\sum F($ fila $A)=0+20+50+110=180$ (suma de los valores dentro de los cuadros).

$\sum \mathrm{F}($ columna $\mathrm{A})=0+0+0+0=0$ Sí. Ir al paso 3.

Paso 2.2. En caso de no poder determinar claramente $D_{1}$ debido a que los dos departamentos que integran $\mathrm{F}_{\mathrm{ij}}{ }^{+}$ tengan la misma área, usar los criterios siguientes para tratar de desempatar. $\sum \mathrm{F}_{\mathrm{D} 1}{ }^{-}=\operatorname{Min}\left(\sum \mathrm{F}_{\mathrm{D} 1, \mathrm{i}^{\prime}}, \sum \mathrm{F}_{\mathrm{D} 1, \mathrm{j}}\right) \mathrm{F}_{\mathrm{D} 2}{ }^{-}=\operatorname{Min}\left(\sum \mathrm{F}_{\mathrm{D} 2, \mathrm{i}^{\prime}} \sum \mathrm{F}_{\mathrm{D} 2, \mathrm{j}}\right) \quad \mathrm{F}^{-}=$ $\operatorname{Max}\left(\sum \mathrm{F}_{\mathrm{D} 1}^{-}, \sum \mathrm{F}_{\mathrm{D} 2}{ }^{-}\right) \cdot$ ¿Se puede definir $\mathrm{D}_{1}$ ?

Sí. Ir al paso 3

No. Ir al paso 2.3

En nuestro caso P1 Forma 1, no aplica.

Paso 2.3. Asignación: Libre elección (romper empates arbitariamente). Ubicación: $\mathrm{d}_{\mathrm{ij}}{ }^{-}$(distancia mínima entre departamentos). En nuestro caso P1 Forma 1, no aplica.

Paso 3. El departamento D1 asignado debe cumplir con los lineamientos de forma sugeridos por las ecuaciones 5, 6 y 7. La longitud del departamento D1 debe estar alineada a la longitud del área disponible, ya que esta alineación asegura que la distancia entre departamentos $\mathrm{d}_{\mathrm{ij}}$ sea mínima.

En nuestro caso P1 Forma 1, el departamento $D_{1}$ asignado debe cumplir con los lineamientos de forma sugeridos por las ecuaciones 5,6 y 7 (tabla 3 y figura 3). La longitud del departamento $\mathrm{D}_{1}=\mathrm{A}$ debe estar alineada con la longitud del área disponible, ya que esta alineación asegura que la distancia entre departamentos $\mathrm{d}_{\mathrm{ij}}$ sea mínima.

Paso 4. Ya asignado $\mathrm{D}_{1}$, se asigna $\mathrm{D}_{2}$ (Departamento no asignado en primer lugar de $\mathrm{Fij}^{+}$) usando los mismos lineamientos de forma usados para alinear $\mathrm{D}_{1}$.

En nuestro caso P1 Forma 1, $\mathrm{D}_{1}=$ Departamento A, se asigna $\mathrm{D}_{2}=\mathrm{D}$ usando los mismos lineamientos de forma usados para alinear $D_{1}$.

Paso 5. Los criterios para asignación a partir del tercer departamento son: $(\mathrm{k} \geq 3)$.

$\mathrm{F}_{\mathrm{k}}^{+}=\operatorname{Max}\left(\mathrm{F}_{\mathrm{ik}}, \mathrm{F}_{\mathrm{jk}}\right) \quad \mathrm{D}_{\mathrm{k}}$ debe estar relacionado con $\mathrm{D}_{1}$ o $\mathrm{D}_{2}$ o hasta $\mathrm{D}_{\mathrm{k}-1}$.

$F_{i, k}=$ Producto costo-flujo del departamento $D_{k}$ por fila 
Tabla 3. Criterios de forma de asignación de departamentos de P1 usando la metodología LDMTP. Fuente: elaboración propia (2016)

\begin{tabular}{|c|c|c|c|c|c|c|c|c|c|c|c|c|c|}
\hline$F_{i j}$ & $\mathrm{D}_{1}$ & $\mathrm{D}_{2}$ & $\mathrm{~A}_{1}$ & $\mathrm{~A}_{2}$ & $\mathrm{~A}^{+}$ & $\sum \mathrm{F}_{\mathrm{ij}}$ & Orden & Depto. & $\mathrm{L}$ & $\mathrm{W}$ & $\mathrm{L} / \mathrm{W}$ & $\mathrm{P}_{\mathrm{i}}$ & $\Omega$ \\
\hline 110 & $\mathrm{~A}$ & $\mathrm{D}$ & 5000 & 5000 & $\mathrm{~A}, \mathrm{D}(5000)$ & $\mathrm{A}(180)$ & $1^{\circ}$ & A & 200 & 25 & $8(+)$ & 450 & 1.59 \\
\hline 50 & A & C & 5000 & 5000 & & & $2^{\circ}$ & D & 200 & 25 & $8(+)$ & 450 & 1.59 \\
\hline 20 & B & $\mathrm{C}$ & 5000 & 5000 & & & $3^{\circ}$ & C & 100 & 50 & $2\left(^{*}\right)$ & 300 & 1.06 \\
\hline 20 & $\mathrm{~A}$ & $\mathrm{~B}$ & 5000 & 5000 & & & $4^{\circ}$ & B & 100 & 50 & $2\left(^{*}\right)$ & 300 & 1.06 \\
\hline
\end{tabular}

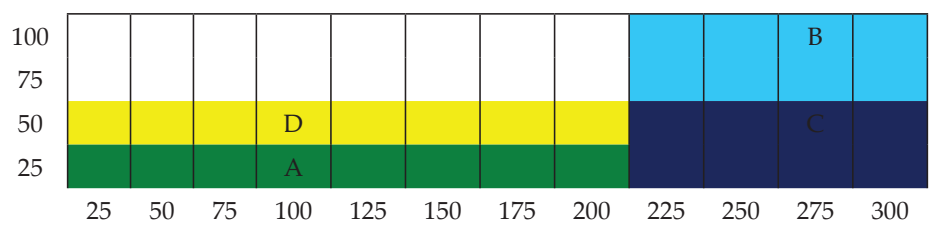

Figura 3. Layout inicial de P1 usando LDMTP

Fuente: elaboración propia (2016)

$\mathrm{F}_{\mathrm{j}, \mathrm{k}}=$ Producto costo-flujo del departamento $\mathrm{D}_{\mathrm{k}}$ por columna

En nuestro caso P1 Forma 1, los criterios para asignación a partir del tercer departamento son que el producto costo-flujo $\mathrm{F}_{\mathrm{D} 3}{ }^{+}=\operatorname{Max}\left(\mathrm{F}_{\mathrm{AC}}, \mathrm{F}_{\mathrm{CA}}, \mathrm{F}_{\mathrm{DC}}, \mathrm{F}_{\mathrm{CD}}, \mathrm{F}_{\mathrm{AB}}, \mathrm{F}_{\mathrm{BA}}, \mathrm{F}_{\mathrm{DB}}\right.$, $\left.\mathrm{F}_{\mathrm{BD}}\right)=\operatorname{Max}(50,0,0,30,20,0,0,10)=50$ que corresponde a $\mathrm{F}_{\mathrm{AC}} \cdot \mathrm{D}_{3}$ debe estar relacionado con $\mathrm{D}_{1}(\mathrm{~A})$ o $\mathrm{D}_{2}(\mathrm{D})$. En este caso $\mathrm{D}_{3}=\mathrm{C}$ se debe alinear con $\mathrm{A}$.

Nota: Estos valores se toman de la tabla 2.

Paso 6. Repetir el procedimiento a partir del paso 5 hasta realizar todas las asignaciones de departamentos tomando en cuenta los lineamientos de forma sugeridos por Bozer et al. (2005).

En nuestro caso P1 Forma 1, $\mathrm{F}_{\mathrm{D} 4}{ }^{+}=\operatorname{Max}\left(\mathrm{F}_{\mathrm{DB}}, \mathrm{F}_{\mathrm{BD}}, \mathrm{F}_{\mathrm{CB}}\right.$, $\left.F_{B C}\right)=\operatorname{Max}(0,10,0,20)=20$, que corresponde a $F_{B C}$. $D_{4}$ está relacionado con $\mathrm{D}_{3}(\mathrm{C})$. En este caso, el cuarto departamento a asignar es $\mathrm{D}_{4}=\mathrm{B}$.

Nota: $\mathrm{D}_{4}$ no puede relacionarse con $\mathrm{D}_{1}$, ya que está bloqueado por $\mathrm{D}_{2}$.

Los datos del problema indican que se cuenta con un área de $\mathrm{L}_{\mathrm{T}}=300 \mathrm{ft} \times \mathrm{W}_{\mathrm{T}}=100 \mathrm{ft}=30,000 \mathrm{ft}^{2}$

$\mathrm{F}_{\mathrm{ij}} \quad=$ Producto costo-flujo de transporte de material unitario entre los departamentos i y j

$\sum \mathrm{F}_{\mathrm{ij}}=$ Sumatoria de $\mathrm{F}_{\mathrm{ij}}$ por fila o columna

$\mathrm{A}_{1}$ = Área del departamento 1, $\mathrm{A}_{2}=$ Área del departa mento $2, \mathrm{~A}^{+}=\mathrm{A}_{\text {mayor }}=5000 \mathrm{y} \mathrm{A}^{-}=\mathrm{A}_{\text {menor }}=5000$, $\mathrm{A}_{\text {mayor }} / \mathrm{A}_{\text {menor }}=1.00$.

Se calcula la relación $\mathrm{L}_{\mathrm{T}} / \mathrm{W}_{\mathrm{T}}$ y de acuerdo con la ecuación 5, Largo $\mathrm{L}_{\mathrm{i}} /$ Ancho $\mathrm{W}_{\mathrm{i}} \geq \mathrm{L}_{\mathrm{T}} / \mathrm{W}_{\mathrm{T}}$ se obtiene lo siguiente:
(+): Los departamentos A y D cumplen con la ecuación 5

$\left(^{*}\right)$ Los departamentos C y B se ajustan a la ecuación 6

El layout inicial se muestra en la figura 3.

Los datos de los centroides (puntos medios) de los departamentos son: $\mathrm{A}\left(\mathrm{X}_{\mathrm{A}}=100\right.$ y $\left.\mathrm{Y}_{\mathrm{A}}=12.5\right), \mathrm{B}\left(\mathrm{X}_{\mathrm{B}}=250, \mathrm{Y}_{\mathrm{B}}\right.$ $=75), C\left(X_{C}=250, Y_{C}=25\right)$ y $D\left(X_{D}=100, Y_{D}=37.5\right)$

$$
\begin{aligned}
& X_{A}=\frac{(0+200)}{2}=100 \quad Y_{A}=\frac{(0+25)}{2}=12.5 \quad X_{D}=\frac{(0+200)}{2}=100 \\
& Y_{D}=\frac{(25+50)}{2}=37.5 \quad X_{C}=\frac{(200+300)}{2}=250 \quad Y_{C}=\frac{(0+50)}{2}=25 \\
& X_{B}=\frac{(200+300)}{2}=250 \quad Y_{B}=\frac{(50+100)}{2}=75
\end{aligned}
$$

La construcción del layout inicial Forma 1 se crea de la siguiente manera:

1. Se asigna el departamento $\mathrm{A}$, ya que da el valor más alto de $\mathrm{F}_{\mathrm{ij}}(110)$ corresponde al intercambio A-D (tabla 4) y también se obtiene el valor más alto de $\sum \mathrm{F}_{\mathrm{ij}}$ = 180 para el departamento A (tabla 4). El departamento A cumple el criterio de la ecuación $5, \mathrm{~L}_{\mathrm{A}} / \mathrm{W}_{\mathrm{A}}=$ $8>3$ (tabla 3 ).

2. Se asigna el departamento $D$, ya que es el segundo valor del intercambio A-D (tabla 4). El departamento $\mathrm{D}$ cumple el criterio de la ecuación $5, \mathrm{~L}_{\mathrm{D}} / \mathrm{W}_{\mathrm{D}}=8>$ 3 (tabla 3).

3. Se asigna el departamento $C$, ya que es el valor remanente más alto de $\mathrm{F}_{\mathrm{ij}}(50)$ y corresponde al intercambio A-C (tabla 4). C debe estar alineado con el departamento A. El departamento C cumple el criterio de la ecuación 6 , (tabla 3). 
4. Se asigna el departamento B, ya que es el valor remanente más alto de $\mathrm{F}_{\mathrm{ij}}(20)$ y corresponde al intercambio B-C o A-B (tabla 4). B puede estar asignado junto al departamento A o departamento $C$, pero como ya no es posible asignar el departamento $B$ junto al departamento A (porque el departamento A está encerrado, ver figura 3), el departamento $B$ debe estar alineado con C. El departamento B departamento C cumple el criterio de la ecuación 6, (tabla 3).

Los centroides de los departamentos $\mathrm{A}\left(\mathrm{X}_{\mathrm{A}}=100\right.$ y $\mathrm{Y}_{\mathrm{A}}=$ 12.5), $B\left(X_{B}=250, Y_{B}=75\right), C\left(X_{C}=250, Y_{C}=25\right)$ y $D\left(X_{D}=\right.$ $\left.100, Y_{D}=37.5\right)$, se usan para calcular las distancias entre departamentos de la forma siguiente

$d_{i j}=\left|X_{i}-X_{j}\right|+\left|Y_{i}-Y_{j}\right|$

La aplicación de la ecuación 10 a los departamentos A, B, C y D es la siguiente:

$\mathrm{d}_{\mathrm{A}-\mathrm{B}}=\left|\mathrm{X}_{\mathrm{A}}-\mathrm{X}_{\mathrm{B}}\right|+\left|\mathrm{Y}_{\mathrm{A}}-\mathrm{Y}_{\mathrm{B}}\right|=|100-250|+|12.5-75|=$ $|-150|+|-62.5|=212.5$, de forma semejante se aplica para conocer las distancias A-C $\left(\mathrm{d}_{\mathrm{A}-\mathrm{C}}=|100-250|+\right.$ $|12.5-25|=162.5)$

A-D $\left(d_{A-D}=|100-100|+|12.5-37.5|=25.0\right), B-C\left(d_{B-C}\right.$ $=|250-250|+|75-25|=50.0)$

B-D $\left(\mathrm{d}_{\mathrm{B}-\mathrm{D}}=|250-100|+|75-37.5|=187.5\right)$ y C-D $\left(\mathrm{d}_{\mathrm{C}-\mathrm{D}}\right.$ $=|250-100|+|25-37.5|=162.5)$.

El costo de la distribución inicial Forma 1 se muestra en la tabla 4.

El costo de la distribución inicial se obtiene de la forma siguiente: $212.5 * \$ 20+162.5 * \$ 50+25.0 * \$ 110+50$ $* \$ 20+187.5 * \$ 10+162.5 * \$ 30=\$ 22,875.00$.

Paso 7. Realizar la distribución inicial de la Forma $1\left(D_{1}\right.$ $\mathrm{D}_{2}, \mathrm{D}_{3}$ ). Determinar el costo total de la primera distribución de planta.
En nuestro caso P1 Forma 1, corresponde a la figura 3 y el costo total de la primera distribución de planta se muestra en la tabla $4(\$ 22,875)$.

Paso 7.1. Si para el tercer departamento a asignar, se cumple que: $\mathrm{D}_{3}$ debe estar junto a $\mathrm{D}_{1}$ y $\mathrm{A}_{\mathrm{D} 3}=\mathrm{A}_{\mathrm{D} 2}=\mathrm{A}_{\mathrm{D} 1}$, se verifica la posibilidad de usar criterios alternativos para asignar el tercer departamento.

\section{Sí. Ir al paso $7.2 \quad$ No. Ir al paso 8}

En nuestro caso P1 Forma 1, el tercer departamento a asignar cumple que: $\mathrm{D}_{3}(\mathrm{C})$ debe estar junto a $\mathrm{D}_{1}(\mathrm{~A})$ y $A_{D 3}=A_{D 2}=A_{D 1}=5,000$, y se debe verificar la posibilidad de usar criterios alternativos para asignar el tercer departamento. Como se cumple, se realiza el intercambio $\mathrm{D}_{2}, \mathrm{D}_{1}, \mathrm{D}_{3}$. Ir al paso 7.2

A partir de este momento, nuestro caso P1 Forma 1 se transforma en P1 Forma 2.

Paso 7.2. Reorganizar el layout de la forma siguiente:

$\mathrm{D}_{\text {1modificado }}=\mathrm{D}_{\text {2original }} \quad \mathrm{D}_{\text {2modificado }}=\mathrm{D}_{\text {1original }}$

$\mathrm{D}_{3 \text { modificado }}=\mathrm{D}_{3 \text { original }}$

Realizar la distribución inicial de la Forma $2\left(\mathrm{D}_{2}, \mathrm{D}_{1}\right.$, $\left.\mathrm{D}_{3}\right)$.

Determinar el costo de la distribución inicial de la Forma 2 y comparar con la Forma 1. ¿\$Forma2 < \$Forma1?

Sí. Ir al paso $7.3 \quad$ No. Ir al paso 8

En nuestro caso P1 Forma 2, $\mathrm{D}_{1 \text { modificado }}=\mathrm{D}_{\text {2original }}=\mathrm{D}$, $\mathrm{D}_{\text {2modificado }}=\mathrm{D}_{\text {1original }}=\mathrm{A}$ y $\mathrm{D}_{3 \text { modificado }}=\mathrm{D}_{\text {3original }}=\mathrm{C}$.

Realizar la distribución inicial de la Forma $2\left(\mathrm{D}_{2}, \mathrm{D}_{1}\right.$, $\left.\mathrm{D}_{3}\right)$, ver tabla 5 y figura 4 .

Tabla 4. Distribución inicial del método LDMTP Forma 1 (Problema 6-Chase et al., 2001). Fuente: elaboración propia (2016)

\begin{tabular}{|c|c|c|c|c|c|c|}
\hline De-Hacia & $\mathbf{A}$ & B & $\mathrm{C}$ & D & & $\sum F_{i j}$ \\
\hline \multirow{2}{*}{$\mathbf{A}$} & 0.0 & 212.5 & 162.5 & 25.0 & 15125,00 & 180 \\
\hline & 0 & 20 & 50 & 110 & $15,125.00$ & 180 \\
\hline \multirow{2}{*}{ B } & 212.5 & 0.0 & 50.0 & 187.5 & 287500 & 30 \\
\hline & $\mathbf{0}$ & 0 & 20 & 10 & $2,0 / 3.00$ & 30 \\
\hline \multirow{2}{*}{ C } & 162.5 & 50.0 & 0.0 & 162.5 & 487500 & 30 \\
\hline & 0 & 0 & 0 & 30 & $4,0 / 2.00$ & 30 \\
\hline \multirow{2}{*}{ D } & 25.0 & 187.5 & 162.5 & 0.0 & 0 & 0 \\
\hline & 0 & 0 & 0 & 0 & 0 & 0 \\
\hline$\sum F_{i j}$ & 0 & 20 & 70 & 150 & $\$ 22,875.00$ & \\
\hline
\end{tabular}


Tabla 5. Criterios de forma de asignación de departamentos de P1Forma 2 usando la metodología LDMTP. Fuente: elaboración propia (2016)

\begin{tabular}{|c|c|c|c|c|c|c|c|c|c|c|c|c|c|c|}
\hline $\mathrm{F}_{\mathrm{ij}}$ & $\mathrm{D}_{1}$ & $\mathrm{D}_{2}$ & $\mathrm{~A}_{1}$ & $\mathrm{~A}_{2}$ & $\mathrm{~A}^{+}$ & $\sum F_{i j}$ & $\mathrm{~d}_{\mathrm{ij}}$ & Orden & Depto. & $\mathrm{L}$ & $\mathrm{W}$ & $\mathrm{L} / \mathrm{W}$ & $P_{i}$ & $\Omega$ \\
\hline 110 & A & $\mathrm{D}$ & 5000 & 5000 & $\mathrm{~A}, \mathrm{D}(5000)$ & $\mathrm{A}(180)$ & & $1^{\circ}$ & A & 200 & 25 & $8(+)$ & 450 & 1.59 \\
\hline 50 & A & $\mathrm{C}$ & 5000 & 5000 & & & & $2^{\circ}$ & D & 200 & 25 & $8(+)$ & 450 & 1.59 \\
\hline 20 & B & $\mathrm{C}$ & 5000 & 5000 & & & 25.0 & $3^{\circ}$ & C & 200 & 25 & $8(+)$ & 300 & 1.06 \\
\hline 20 & A & B & 5000 & 5000 & & & 162.5 & $4^{\circ}$ & B & 200 & 25 & $8(+)$ & 300 & 1.06 \\
\hline
\end{tabular}

$\mathrm{F}_{\mathrm{ij}} \quad=$ Producto costo-flujo de transporte de material unitario entre los departamentos i y j

$\sum \mathrm{F}_{\mathrm{ij}}=$ Sumatoria de $\mathrm{F}_{\mathrm{ij}}$ por fila o columna

$\mathrm{A}_{1}$ = Área del departamento 1, $\mathrm{A}_{2}=$ Área del departamento $2, \mathrm{~A}^{+}=\mathrm{A}_{\text {mayor }}=5000 \mathrm{y} \mathrm{A}^{-}=\mathrm{A}_{\text {menor }}=5000$ $\mathrm{A}_{\text {mayor }} / \mathrm{A}_{\text {menor }}=1.00$.

Se calcula la relación $\frac{\mathrm{L}_{\mathrm{T}}}{\mathrm{W}_{\mathrm{T}}}=\frac{300}{100}=3 \mathrm{y}$ de acuerdo con la ecuación 5, $\frac{\mathrm{L} \arg 0 \mathrm{~L}_{\mathrm{i}}}{\text { Ancho } \mathrm{W}_{\mathrm{i}}} \geq \frac{\mathrm{L}_{\mathrm{T}}}{\mathrm{W}_{\mathrm{T}}}$ se obtiene lo siguiente:

(+): Los departamentos A, B, C y D cumplen con la ecuación 5 .

El layout inicial de la Forma 2 se muestra en la figura 4.

Los datos de los centroides (puntos medios) de los departamentos son: $\mathrm{A}\left(\mathrm{X}_{\mathrm{A}}=100\right.$ y $\left.\mathrm{Y}_{\mathrm{A}}=37.5\right), \mathrm{B}\left(\mathrm{X}_{\mathrm{B}}=100\right.$, $\left.\mathrm{Y}_{\mathrm{B}}=87.5\right), \mathrm{C}\left(\mathrm{X}_{\mathrm{C}}=100, \mathrm{Y}_{\mathrm{C}}=62.5\right)$ y $\mathrm{D}\left(\mathrm{X}_{\mathrm{D}}=100, \mathrm{Y}_{\mathrm{D}}=12.5\right)$

$X_{A}=\frac{(0+200)}{2}=100 \quad Y_{A}=\frac{(25+50)}{2}=37.5 \quad X_{D}=\frac{(0+200)}{2}=100$

$Y_{D}=\frac{(0+25)}{2}=12.5 \quad X_{C}=\frac{(0+200)}{2}=100 \quad Y_{C}=\frac{(50+75)}{2}=62.5$

$X_{B}=\frac{(0+200)}{2}=100 \quad Y_{B}=\frac{(75+100)}{2}=87.5$

La construcción del layout inicial Forma 2 se muestra de la siguiente manera:

1. Se reasigna el departamento $D$, ya que es el segundo valor del intercambio A-D (tabla 4). El departamento $\mathrm{D}$ cumple el criterio de la ecuación $5, \mathrm{~L}_{\mathrm{D}} / \mathrm{W}_{\mathrm{D}}=8>$ 3 (tabla 5).
2. Se asigna el departamento A como el segundo departamento. El departamento D cumple el criterio de la ecuación $5, \mathrm{~L}_{\mathrm{D}} / \mathrm{W}_{\mathrm{D}}=8>3$ (tabla 5).

3. Se asigna el departamento $C$, ya que es el valor remanente más alto de $\mathrm{F}_{\mathrm{ij}}(50)$ y corresponde al intercambio A-C (tabla 5). C debe estar alineado con el departamento A. El departamento C cumple el criterio de la ecuación $5, \mathrm{~L}_{\mathrm{C}} / \mathrm{W}_{\mathrm{C}}=8>3$ (tabla 5).

4. Se asigna el departamento $B$, ya que es el valor remanente más alto de $\mathrm{F}_{\mathrm{ij}}(20)$ y corresponde al intercambio B-C o A-B (tabla 5). B puede asignarse junto al departamento A o departamento C, pero como ya no es posible asignar el departamento B junto al departamento A (porque el departamento A está encerrado, ver figura 3), el departamento B debe estar alineado con $\mathrm{C}$. El departamento B cumple el criterio de la ecuación $5, \mathrm{~L}_{\mathrm{B}} / \mathrm{W}_{\mathrm{B}}=8>3$ (tabla 5).

Con los datos de los centroides de los departamentos $\mathrm{A}$ $\left(X_{\mathrm{A}}=100\right.$ y $\left.\mathrm{Y}_{\mathrm{A}}=37.5\right), \mathrm{B}\left(\mathrm{X}_{\mathrm{B}}=250, \mathrm{Y}_{\mathrm{B}}=87.5\right), \mathrm{C}\left(\mathrm{X}_{\mathrm{C}}=100\right.$, $\left.\mathrm{Y}_{\mathrm{C}}=62.5\right)$ y $\mathrm{D}\left(\mathrm{X}_{\mathrm{D}}=100, \mathrm{Y}_{\mathrm{D}}=12.5\right)$, se calculan las distancias entre departamentos con la ecuación 10.

La aplicación de la ecuación 10 a los departamentos A, B, C y D es la siguiente:

$\mathrm{d}_{\mathrm{A}-\mathrm{B}}=\left|\mathrm{X}_{\mathrm{A}}-\mathrm{X}_{\mathrm{B}}\right|+\left|\mathrm{Y}_{\mathrm{A}}-\mathrm{Y}_{\mathrm{B}}\right|=|100-100|+|37.5-87.5|$ $=|0|+|-50|=50.0$, de forma semejante se aplica para conocer las distancias A-C $\left(\mathrm{d}_{\mathrm{A}-\mathrm{C}}=|100-100|+\mid 37.5-\right.$ $62.5 \mathrm{I}=25.0)$

A-D $\left(\mathrm{d}_{\mathrm{A}-\mathrm{D}}=|100-100|+|37.5-12.5|=25.0\right), \mathrm{B}-\mathrm{C}\left(\mathrm{d}_{\mathrm{B}-\mathrm{C}}\right.$ $=100-100|+| 87.5-62.5 \mid=25.0)$

B-D $\left(\mathrm{d}_{\text {B-D }}=|100-100|+|87.5-12.5|=75.0\right)$ y C-D $\left(\mathrm{d}_{\text {C-D }}\right.$ $=|100-100|+|62.5-12.5|=50.0)$.

La distribución inicial con intercambio de departamentos $\left(D_{2}, D_{1}, D_{3}\right)$ se muestra en la tabla 6 .

Figura 4. Layout inicial de P1 Forma 2 usando LDMTP. Fuente: elaboración propia (2016)

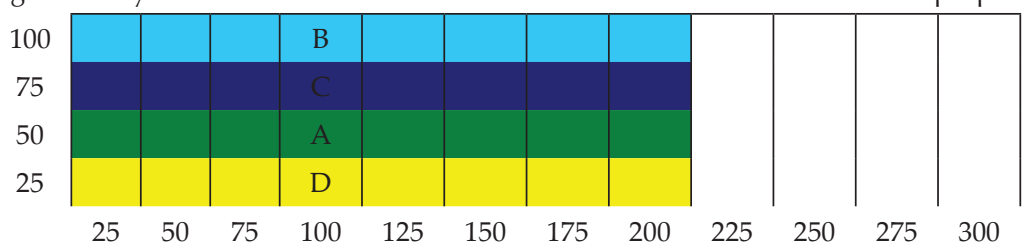


El costo de la distribución inicial se obtiene de la forma siguiente: $50 * \$ 20+25.0 * \$ 50+25.0 * \$ 110+25 * \$ 20+$ $75 * \$ 10+50 * \$ 30=\$ 7,750.00$

En nuestro caso P1 Forma 2, se determina el costo de la distribución inicial de la Forma $2(\$ 7,750)$ y se compara con la Forma 1. ¿\$Forma2 < \$Forma1? $(\$ 7,750<$ $\$ 22,875)$. La respuesta es sí. Ir al paso 7.3.

Paso 7.3 Hacer todo el análisis posterior con la Forma 2.

Paso 8. Establecer todos los intercambios válidos entre dos departamentos (Nahmias, 1999) que pueden ser:

a) Que los dos departamentos a intercambiar tengan una frontera en común.

b) Que los dos departamentos a intercambiar tengan la misma área, aunque no sean contiguos.

Nota: El intercambio que se realizó para generar un nuevo layout, no es un intercambio válido para esa nueva distribución, pero sí para distribuciones siguientes. Por ejemplo, si el intercambio A-B es el origen del $2^{\circ}$ layout (no es un intercambio válido para este layout), pero sí puede considerarse un intercambio válido a partir del 3er layout (siempre y cuando se cumpla alguna de las condiciones mencionadas en el paso 8).
En nuestro caso P1 Forma 2, establece todos los intercambios válidos entre dos departamentos, de acuerdo con la figura 4, que son A-B, A-D y B-C (inciso a, tienen una frontera en común); A-B, B-D y C-D (inciso b, tienen la misma área, aunque no sean contiguos).

Paso 9. Realizar todos los intercambios válidos entre dos departamentos (intercambio de coordenadas: criterio sugerido por CRAFT). Determinar si existe un intercambio que pudiera reducir el costo total de la distribución de planta. ¿Existe un intercambio para el cual el costo del intercambio < costo del origen?

Sí. Ir al paso 10

No. Esta es la distribución de planta (layout) óptima

En nuestro caso P1 Forma 2, se realizan los intercambios válidos: A-B, A-C, A-D, B-C, B-D y C-D. Las diferencias se calculan de la forma siguiente: $(A-B)=$ $\$ 12,750.00-\$ 7,750.00$ (Origen) $=\$ 5,000.00$, de forma semejante se obtienen las diferencias A-C (\$9,750 $\$ 7,750=\$ 2,000)$, A-D $(\$ 8,500-\$ 7,750=\$ 750)$, B-C $(\$ 9,000$ - $\$ 7,750=\$ 1,250), \mathrm{B}-\mathrm{D}(\$ 9,750-\$ 7,750=\$ 2,000.00) \mathrm{y}$ C-D $(\$ 8,250-\$ 7,750=\$ 500)$. Los resultados de los costos obtenidos de los intercambios válidos se muestran en la tabla 7.

Tabla 6. Distribución inicial del método LDMTP Forma 2 P1 (Problema 6-Chase et al., 2001).

Fuente: elaboración propia (2016)

\begin{tabular}{|c|c|c|c|c|c|c|}
\hline De-Hacia & A & B & C & D & & $\sum F_{i j}$ \\
\hline \multirow[b]{2}{*}{$\mathbf{A}$} & 0.0 & 50.0 & 25.0 & 25.0 & \multirow{2}{*}{$5,000.00$} & \multirow{2}{*}{180} \\
\hline & 0 & 20 & 50 & 110 & & \\
\hline \multirow{2}{*}{ B } & 50.0 & 0.0 & 25.0 & 75.0 & \multirow{2}{*}{$1,250.00$} & \multirow{2}{*}{30} \\
\hline & 0 & 0 & 20 & 10 & & \\
\hline \multirow{2}{*}{ C } & 25.0 & 25.0 & 0.0 & 50.0 & \multirow{2}{*}{00} & \multirow{2}{*}{30} \\
\hline & 0 & 0 & 0 & 30 & & \\
\hline \multirow[b]{2}{*}{ D } & 25.0 & 75.0 & 50.0 & 0.0 & \multirow{2}{*}{0.00} & \multirow{2}{*}{$\mathbf{0}$} \\
\hline & 0 & 0 & 0 & 0 & & \\
\hline$\sum F_{i j}$ & 0 & 20 & 70 & 150 & $\$ 7,750.00$ & \\
\hline
\end{tabular}


Tabla 7. Intercambios válidos de P1 Forma 2 (Problema 6-Chase et al., 2001). Fuente: elaboración propia (2016)

\begin{tabular}{|c|c|c|c|c|c|c|c|}
\hline Intercambio & Centroides & & & & stribucic & & \\
\hline \multirow{9}{*}{$\begin{array}{c}\mathrm{A}-\mathrm{B} \\
\mathrm{A}_{\mathrm{A}}=5,000 \\
\mathrm{~A}_{\mathrm{B}}=5,000 \\
\mathrm{~A}_{\mathrm{C}}=5,000 \\
\mathrm{~A}_{\mathrm{D}}=5,000 \\
\mathrm{~A}^{+} / \mathrm{A}^{-}=1.00\end{array}$} & \multirow{10}{*}{$\begin{array}{l}X_{A}=100.0, Y_{A}=87.5 \\
X_{B}=100.0, Y_{B}=37.5 \\
X_{C}=100.0, Y_{C}=62.5 \\
X_{D}=100.0, Y_{D}=12.5\end{array}$} & De-Hacia & \multirow{2}{*}{$\begin{array}{c}1 \\
0.00\end{array}$} & \multirow{2}{*}{$\begin{array}{c}2 \\
50.00\end{array}$} & \multirow{2}{*}{$\begin{array}{c}3 \\
25.00\end{array}$} & \multirow{2}{*}{$\begin{array}{c}4 \\
7500\end{array}$} & \multirow{3}{*}{10500.00} \\
\hline & & \multirow{2}{*}{1} & & & & & \\
\hline & & & \multirow{2}{*}{$\begin{array}{rl}0 & 0 \\
5000 & \end{array}$} & 20 & 50 & 110 & \\
\hline & & \multirow[b]{2}{*}{2} & & 0.00 & 25.0 & 25.00 & \multirow{2}{*}{750.00} \\
\hline & & & 0 & 0 & 20 & 10 & \\
\hline & & & 25.00 & 25.00 & 0.00 & 50.00 & 150000 \\
\hline & & 3 & 0 & 0 & 0 & 30 & 1500.00 \\
\hline & & 4 & 75.00 & 25.00 & 50.00 & 0.00 & 000 \\
\hline & & & 0 & 0 & 0 & 0 & 0.00 \\
\hline & & & & & & & $12,750.00$ \\
\hline & & De-Hacia & 1 & 2 & 3 & 4 & \\
\hline & & & 0.00 & 25.00 & 25.00 & 50.00 & \\
\hline $\mathrm{A}-\mathrm{C}$ & & 1 & 0 & 20 & 50 & 110 & 7250.00 \\
\hline$A_{A}=5,000$ & $\mathrm{X}_{\mathrm{A}}=100.0, \mathrm{Y}_{\mathrm{A}}=62.5$ & 2 & 25.00 & 0.00 & 50.0 & 75.00 & 175000 \\
\hline $\mathrm{A}_{\mathrm{B}}=5,000$ & $X_{B}=100.0, Y_{B}=87.5$ & 2 & 0 & 0 & 20 & 10 & 1750.00 \\
\hline $\mathrm{A}_{\mathrm{C}}=5,000$ & $X_{C}=100.0, Y_{C}=37.5$ & 3 & 25.00 & 50.00 & 0.00 & 25.00 & 750.00 \\
\hline$A_{D}=5,000$ & $X_{D}=100.0, Y_{D}=12.5$ & 3 & 0 & 0 & 0 & 30 & 150.00 \\
\hline $\mathrm{A}^{+} / \mathrm{A}^{-}=1.00$ & & 4 & 50.00 & 75.00 & 25.00 & 0.00 & 0.00 \\
\hline & & & 0 & 0 & 0 & 0 & $9,750.00$ \\
\hline & & De-Hacia & 1 & 2 & 3 & 4 & \\
\hline & & & 0.00 & 75.00 & 50.00 & 25.00 & \\
\hline A-D & & 1 & 0 & 20 & 50 & 110 & 6750.00 \\
\hline $\mathrm{A}_{\mathrm{A}}=5,000$ & $\mathrm{X}_{\mathrm{A}}=100.0, \mathrm{Y}_{\mathrm{A}}=12.5$ & 2 & 75.00 & 0.00 & 25.0 & 50.00 & 100000 \\
\hline $\mathrm{A}_{\mathrm{B}}=5,000$ & $X_{B}=100.0, Y_{B}=87.5$ & 2 & 0 & 0 & 20 & 10 & 1000.00 \\
\hline $\mathrm{A}_{\mathrm{C}}=5,000$ & $X_{C}=100.0, Y_{C}=62.5$ & 3 & 50.00 & 25.00 & 0.00 & 25.00 & 75000 \\
\hline$A_{D}=5,000$ & $X_{D}=100.0, Y_{D}=37.5$ & & 0 & 0 & 0 & 30 & 150.00 \\
\hline $\mathrm{A}^{+} / \mathrm{A}^{-}=1.00$ & & 4 & 25.00 & 50.00 & 25.00 & 0.00 & 0.00 \\
\hline & & & & 0 & 0 & 0 & $8,500.00$ \\
\hline & & De-Hacia & 1 & 2 & 3 & 4 & \\
\hline & & & 0.00 & 25.00 & 50.00 & 25.00 & \\
\hline $\mathrm{B}-\mathrm{C}$ & & 1 & 0 & 20 & 50 & 110 & 5750.00 \\
\hline$A_{A}=5,000$ & $\mathrm{X}_{\mathrm{A}}=100.0, \mathrm{Y}_{\mathrm{A}}=37.5$ & & 25.00 & 0.00 & 25.0 & 50.00 & \\
\hline $\mathrm{A}_{\mathrm{B}}=5,000$ & $X_{B}=100.0, Y_{B}=62.5$ & 2 & 0 & 0 & 20 & 10 & 1000.00 \\
\hline$A_{C}=5,000$ & $X_{C}=100.0, Y_{C}=87.5$ & 3 & 50.00 & 25.00 & 0.00 & 75.00 & 225000 \\
\hline$A_{D}=5,000$ & $X_{D}=100.0, Y_{D}=12.5$ & 3 & 0 & 0 & 0 & 30 & 2250.00 \\
\hline $\mathrm{A}^{+} / \mathrm{A}^{-}=1.00$ & & 4 & 25.00 & 50.00 & 75.00 & 0.00 & (200 \\
\hline & & & 0 & 0 & 0 & 0 & 0.00 \\
\hline & & & & & & & $9,000.00$ \\
\hline & & De-Hacia & 1 & 2 & 3 & 4 & \\
\hline & & 1 & 0.00 & 25.00 & 25.00 & 50.00 & 725000 \\
\hline B-D & & & 0 & 20 & 50 & 110 & 7250.00 \\
\hline$A_{A}=5,000$ & $\mathrm{X}_{\mathrm{A}}=100.0, \mathrm{Y}_{\mathrm{A}}=37.5$ & & 25.00 & 0.00 & 50.0 & 75.00 & 175000 \\
\hline$A_{B}=5,000$ & $X_{B}=100.0, Y_{B}=12.5$ & 2 & 0 & 0 & 20 & 10 & 1750.00 \\
\hline $\mathrm{A}_{\mathrm{C}}=5,000$ & $X_{C}=100.0, Y_{C}=62.5$ & & 25.00 & 50.00 & 0.00 & 25.00 & \\
\hline$A_{D}=5,000$ & $X_{D}=100.0, Y_{D}=87.5$ & 3 & 0 & 0 & 0 & 30 & 750.00 \\
\hline $\mathrm{A}^{+} / \mathrm{A}^{-}=1.00$ & & 4 & 50.00 & 75.00 & 25.00 & 0.00 & החم \\
\hline & & & 0 & 0 & 0 & 0 & 0.00 \\
\hline & & & & & & & $9,750.00$ \\
\hline & & De-Hacia & 1 & 2 & 3 & 4 & \\
\hline & & & 0.00 & 50.00 & 25.00 & 25.00 & \\
\hline C-D & & 1 & 0 & 20 & 50 & 110 & 5000.00 \\
\hline $\mathrm{A}_{\mathrm{A}}=5,000$ & $\mathrm{X}_{\mathrm{A}}=100.0, \mathrm{Y}_{\mathrm{A}}=37.5$ & 2 & 50.00 & 0.00 & 75.0 & 25.00 & 1750.00 \\
\hline$A_{B}=5,000$ & $X_{B}=100.0, Y_{B}=87.5$ & 2 & 0 & 0 & 20 & 10 & 1750.00 \\
\hline $\mathrm{A}_{\mathrm{C}}=5,000$ & $X_{C}=100.0, Y_{C}=12.5$ & 3 & 25.00 & 75.00 & 0.00 & 50.00 & 1500.00 \\
\hline$A_{D}=5,000$ & $X_{D}=100.0, Y_{D}=62.5$ & & 0 & 0 & 0 & 30 & \\
\hline $\mathrm{A}^{+} / \mathrm{A}^{-}=1.00$ & & 4 & $\begin{array}{r}25.00 \\
0\end{array}$ & $\begin{array}{r}25.00 \\
0\end{array}$ & $\begin{array}{r}50.00 \\
0\end{array}$ & $\begin{array}{c}0.00 \\
0\end{array}$ & 0.00 \\
\hline
\end{tabular}


Nota: Como todos los intercambios son mayores que el origen $(>\$ 7,750)$, esto significa que este es el layout óptimo y, por lo tanto, no se aplican los pasos 10 y 11.

Paso 10. Modificar el layout, recalcular los centroides y determinar el costo del origen del nuevo layout.

Nota: Si un departamento no tiene una forma rectangular o cuadrada, es necesario aplicar las fórmulas para el cálculo de centroides (Nahmias 1999) mostradas en la ecuaciones 8 y 9 .

$M_{x}=\frac{\sum_{i=1}^{i=k}\left[\left(X_{2 i}{ }^{2}-X_{1 i}{ }^{2}\right)\left(Y_{2 i}-Y_{1 i}\right)\right]}{2}$

$M_{y}=\frac{\sum_{i=1}^{i=k}\left[\left(Y_{2 i}{ }^{2}-Y_{1 i}{ }^{2}\right)\left(X_{2 i}-X_{1 i}\right)\right]}{2}$

$\bar{X}=\frac{M_{x}}{A} \quad \bar{Y}=\frac{M_{y}}{A}$

En nuestro caso P1 Forma 2, no aplica.

Paso 11. Repetir los pasos 8 al 10 (de ser necesario).

En nuestro caso P1 Forma 2, no aplica.

\section{Prueba de normalidad y test de prueba estadístico}

El test de prueba estadístico empleado para validar los resultados obtenidos, depende de la aplicación de la prueba de la normalidad llamada prueba de Anderson Darling (Kvam y Vidakovic 2007), para los cálculos se empleó el "Menú Estadísticas básicas Prueba de normalidad Anderson-Darling" de MINITAB 17. Las hipótesis son:

$\mathrm{H}_{0}$ : Los datos se ajustan a una distribución normal. $\mathrm{H}_{\mathrm{a}}$ : Los datos no se ajustan a una distribución normal.

\section{Criterio de decisión}

La hipótesis nula $\left(\mathrm{H}_{0}\right)$ se rechaza con un nivel de significancia $\alpha$ si $\mathrm{A}^{2^{*}}>0.752(\alpha=0.05)$ y si $\mathrm{P}<0.05$ (Anholeto 2007). Si se acepta $H_{0}$ se aplica el test " $t$ de muestras dependientes", si se rechaza $\mathrm{H}_{0}$, se usa la "Prueba $\mathrm{Z}$ de los rangos con signo" (Weimer 1996), figura 5.

\section{Prueba de los rangos con signo}

$\mu_{\mathrm{T}}=$ Promedio de los $n$ casos

$\sigma_{\mathrm{T}}=$ Desviación es tan dar de los $n$ casos

$\mathrm{Z}_{\mathrm{T}}=$ Estadística de prueba

$$
\mu_{T}=\frac{n(n+1)}{4} \sigma_{T}=\sqrt{\frac{n(n+1)(2 n+1)}{24}} \quad Z=\frac{T-\mu_{T}}{\sigma_{T}} n \geq 15
$$

Tabla 8. Datos sintetizados de 50 casos usando valor final de costo (MFFC).

Fuente: elaboración propia (2016)

\begin{tabular}{cccc}
\hline & \multicolumn{3}{c}{ Valor final de costo MFFC $(\mathrm{n}=50)$} \\
\hline & LDMTP & CRAFT & Diferencia \\
Promedio & $25,254.67$ & $30,391.30$ & $-5,136.63$ \\
Desviación estándar (s) & $80,894.3987$ & $94,290.4394$ & \\
\hline
\end{tabular}

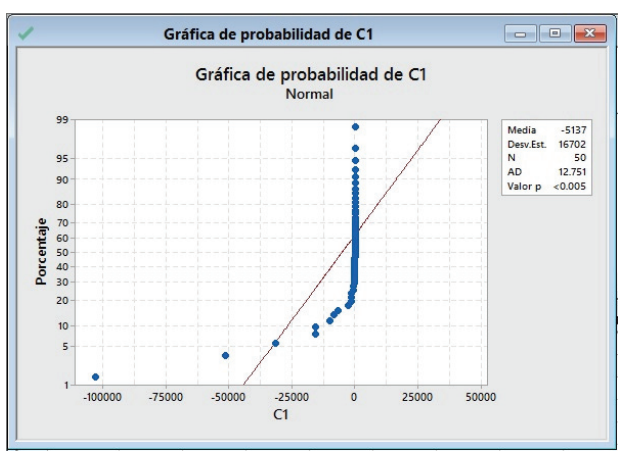

Figura 5. Análisis de Anderson-Darling de MFFC. Fuente: Elaboración propia (2016)
Los resultados de Anderson Darling son: $\mathrm{A}^{2^{*}}=12.751\left(\mathrm{~A}^{2^{*}}>0.752\right)$ y $\mathrm{p}=$ $0.0000(\mathrm{p}<0.05)$, se rechaza $\mathrm{H}_{0}$ y se acepta $\mathrm{H}_{\mathrm{a}^{\prime}}$ los datos no proceden de una distribución normal y se utiliza la prueba "De los rangos con signo". 
$S=$ Suma de rangos $=\mathrm{T}^{+}+\mathrm{T}^{-}$

$\mathrm{H}_{0}$ : LDMTP da peores resultados o ambos métodos dan igual resultado $\left(\mu_{\mathrm{LDMTP}}-\mu_{\mathrm{CRAFT}} \geq 0\right)$.

$\mathrm{H}_{\mathrm{a}}$ : LDMTP da mejores resultados $\left(\mu_{\mathrm{LDMTP}}-\mu_{\mathrm{CRAFT}}<0\right)$.

Criterio de decisión: Si $Z \geq Z_{\alpha}$ se rechaza $\mathrm{H}_{\mathrm{O}}$ y como $\alpha=0.05$, se convierte en $Z \geq Z_{0.05}$ ( $Z \geq 1.645$ valor obtenido de tabla de distribución normal).
La prueba de los rangos con signo de Wilcoxon para el valor final de costo (MFFC) se realizó calculando las diferencias de MFFC (LDMTP-CRAFT) y usando el software estadístico MINTAB 17 con la prueba Estadísticas No paramétricos de "Wilcoxon de 1 muestra" y se observa el procedimiento de cálculo en las figuras 6 a 10.

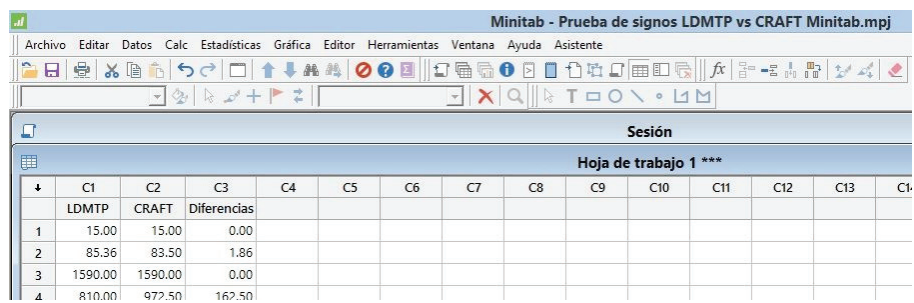

Figura 6. Alimentación de datos de MFFC de LDMTP, CRAFT y sus diferencias

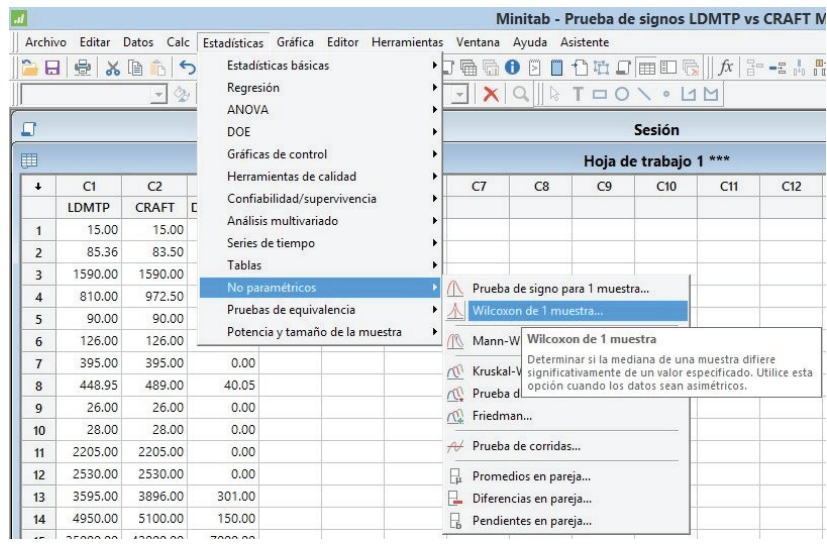

Figura 7. Selección del Menú Estadísticas No paramétricos Wilcoxon de 1 muestra

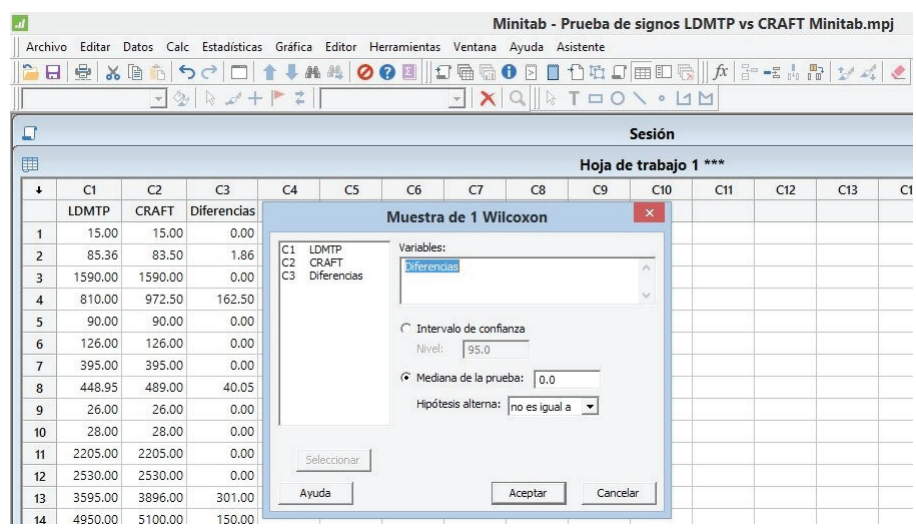

Figura 8. Selección de la variable a analizar (Diferencias) y el tipo de Estadístico (Mediana)
Fuente: elaboración propia (2016) 
Prueba de la mediana $=0.000000$ vs. la mediana $\neq 0.000000$

\begin{tabular}{|c|c|c|c|c|c|}
\hline & & $\begin{array}{r}\text { Número } \\
\text { de }\end{array}$ & Estadística & & $\mathrm{Me}$ \\
\hline 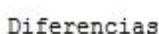 & $\begin{array}{r}\text { N } \\
50\end{array}$ & 38 & 741.0 & 0.000 & 352.5 \\
\hline
\end{tabular}

Figura 9. Resultados globales (S).

Fuente: elaboración propia (2016)

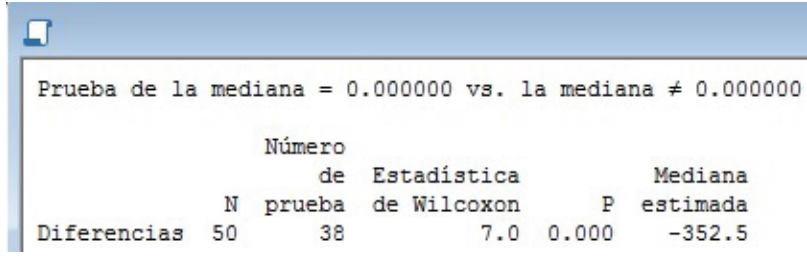

Figura 10. Resultados de T+

Fuente: elaboración propia (2016)

El análisis de las figuras 6 a 10 muestra los resultados siguientes:

Nota: Para asignar la posición relativa se excluyen los ceros $\left(0^{\prime} \mathrm{s}\right), \mathrm{n}=50-12=38$.

Las fórmulas y resultados de la Prueba de los Rangos con signo se muestran a continuación:

$$
\begin{aligned}
\mu_{T}= & \frac{n(n+1)}{4}=\frac{38(38+1)}{4}=\frac{38(39)}{4}=370.5 \\
\sigma_{T}= & \sqrt{\frac{n(n+1)(2 n+1)}{24}}=\sqrt{\frac{38(38+1)(2 * 38+1)}{24}}= \\
& \sqrt{\frac{38(39)(77)}{24}}=68.9547
\end{aligned}
$$

$\mathrm{S}=$ Suma de rangos $=\mathrm{T}^{+}+\mathrm{T}^{-}=741.0$

$\mathrm{T}^{+}=7.0 \mathrm{~T}^{-}=\mathrm{S}-\mathrm{T}^{+}=741.0-7.0$

$\mathrm{T}^{\mathrm{N}}=\mathrm{T}=734.0$

$\mathrm{Z}=\frac{T-\mu_{T}}{\sigma_{T}}=\frac{T^{-}-\mu_{T}}{\sigma_{T}}=\frac{734.0-370.5}{68.9547}=5.2716$

Como $Z>Z_{0.05}(5.2716>1.6450)$ se rechaza Ho y se acepta $\mathrm{Ha}$, es decir, que LDMTP produce menores valores finales de costo (MFFC) que CRAFT.

\section{Conclusiones}

La novedad que aporta esta heurística es que hasta ahora no se ha encontrado en la literatura ninguna aplicación del diseño óptimo de la distribución de planta (ODPL) basada en el problema de transporte. El proceso de es- tablecer la asignación inicial de la heurística LDMTP no es un asunto subjetivo, debido a que la elección del mejor orden de asignación de los departamentos que conforman la distribución inicial sigue una serie de pasos y tiene hasta 4 diferentes criterios de desempate, lo cual favorece que se elija el arreglo de departamentos que puede aportar la mayor contribución a la reducción del costo total de la distribución

La heurística LDMTP ha demostrado que es eficiente para resolver OPDL, debido a que este es un método selectivo capaz de distinguir el mejor orden de asignación de todos los departamentos que conforman el layout, incluyendo criterios de forma (largo y ancho) para cada departamento asignado. Esta información se ve claramente reflejada con los resultados obtenidos para el análisis de 50 casos referenciados en la literatura, para el valor final que se expresa mediante las sumas de las características donde LDMTP obtuvo un valor final de $16.90 \%$ menor que con CRAFT.

Estadísticamente se demuestra mediante el uso de la "Prueba $Z$ de los rangos con signo" que el método LDMTP es mejor que CRAFT, que arrojó el resultado siguiente, Valor final $Z>Z_{0.05}(5.2716>1.6450)$, lo que significa que LDMTP es más eficiente y produce menores valores de costo (MFFC) que CRAFT.

\section{Agradecimientos}

El autor desea agradecer al personal de la UTSC, al rector Dr. José Cárdenas Cavazos y la Directora Académica Mtra. Ana Bertha Tamez Salas por las facilidades otorgadas para realizar este trabajo y a los MES. Manuel Cantú Sosa y Anselmo Carpio Hernández, del cuerpo académico de procesos industriales por sus sugerencias de mejora del método.

\section{Referencias}

Adi A. et al. Slideshare [en línea] 30 de junio de 2013 [Fecha de consulta: 14 de octubre de 2014]. Disponible en: http://www. slideshare.net/renspady/craft-software-for-dummies.

Anholeto A. et al. Abordagem estatística na validação retrospectiva do processo de fabricação de mistura polivitamínica. Revista Brasileira de Ciências Farmacêuticas, volumen 43 (número 2), junio de 2007: 10.

Bozer Y., Meller R., Erlebacher S.. University of Michigan Library [en línea] 15 de marzo de 2005 [Fecha de consulta: 14 de octubre de 2014]. Disponible en: deepblue.lib.umich.edu/bitstream/handle/.../ban1374.0001.001.pdf?...5.

Chary S. Theory and problems in production and operations management, ed. Tata Mc Graw Hill, Nueva Delhi, Tata Mc Graw Hill, 1995, pp. 90-129. 
Chase R., Aquilano N., Jacobs F. Operations management for competitive advantage, Nueva York, Mc Graw Hill, 2001, pp. 189214.

Galindo A. y Tapia M. SPL: Una forma sencilla de analizar la distribución física de su fábrica, 2, Habana, CUJAE, Ingeniería Industrial, volumen 29, 2013: 1-6.

Heizer R. y Render B. Operations management, Upper Saddle River, Prentice Hall, 2001, pp. 340, 362-369.

Hillier F. y Lieberman G. Introductions to Operations Research, 7a ed., Nueva York, Mc Graw Hill, 2001.

Jithavech I. y Kumar K. A simulation-based approach for risk assesssment of facility layout design under stochastics product demands, 49, Cham: IJAMT. International Journal of advanced Manufacturing Technology, volumen 1, julio de 2010: 27-40.

John B., James J., Rengaraj R. Analysis and optimization of plant layout using relative allocation of facilities technique. 8, Nueva Delhi, IJETAE. Internattional Journal of Emerging Technology and Advanced Engineering, volumen 3, agosto de 2013: 514-520.

Kvam P. y Vidakovic B. Nonparametric statistics with applications to science and engineering, Hoboken, John Wiley \& Sons, Inc., 2007, pp. 90.

Mejía C. Universidad Nacional de Colombia [en línea] 2 de Octubre de 2012 [Fecha de consulta: 14 de Octubre de 2014]. Disponibleen:www.bdigital.unal.edu.co/.../camilomejiamoncayo.20...

Nahmias S. Análisis de la produción y las operaciones, 1a. ed., México, CECSA, 1999, pp. 558-561,567-569,595-596.

Wang M., Hu M., Ku M. A solution to unequal area facilities layout problem by genetic algorithm, Gronigen, Elsevier. Computers in Industry, volumen 56, febrero de 2005: 207-220.

Weimer R. Estadística, 1ํㅡㄹ ed., México, CECSA, 1996, pp. 537, 723.

\section{Bibliografía}

Cobo A. y Serrano A. Revista virtual Pro [en línea] junio de 2011 [citado: 14 de octubre de 2014]. Disponible en: http://www. revistavirtualpro.com/revista/distribucion-de-planta/22\#1555.

Chen C. Power show [en línea] [Fecha de consulta: 14 de octubre de 2014]. Disponible en: http://www.powershow.com/
view/2712d2-MzQ5M/Chapter_6_Layout_Planning_Models_ and_Design_Algorithms_powerpoint_ppt_presentation.

Cherbaka N. Universidad ICESI [en línea] 17 de marzo de 2006 [Fecha de consulta: 14 de octubre de 2014]. Disponible en: ftp://ftp.icersi.edu.co/leonardo/DistPlanta/Slides/Layout-4-05166.ppt.

Fazle M. [en línea] 7 de noviembre de 1999 [Fecha de consulta: 14 de octubre de 2014]. Disponible en: web4.uwindsor.ca/.../ baki\%20fazle/.../Chapter_10_Lecture_20_to_23_2....

Jensen P. y Bard J. [en línea] 7 de enero de 2012 [Fecha de consulta: 9 de octubre de 2014]. Disponible en: busieco.samnet.sdu.dk/ undervis/90336.E05/FacLayoutfrajensen.pdf.

Kumar S. National Institute of Technology Rourkela [en línea] 6 de junio de 2007 [Fecha de consulta: 14 de octubre de 2014]. Diponible en: ethesis.nitrkl.ac.in/4348/1/Design_of_Plant.pdf.

Lepcha G. y Roy D. Job Shop Layout Design Using Group Technology, 3, Washington, IJMER, International Journal of Modern Engineering Research, volumen 3, mayo de 2013: 1268-1272.

Mukhopaday S. Production Planning Text and cases, Nueva Delhi, ed. PHI Learning Private Limited, PHI Learning Private Limited, 2009, pp. 13-24.

Muñoz M. Universidad Nacional Mayor de San Marcos [en línea] 2004 [Fecha de consulta: 9 de octubre de 2014]. Disponible en: sisbib.unmsm.edu.pe > Colección digital.

Narayana S., Varaprasad V., Veeranna V. Optimization of multiobjectve facility layout using non-traditional optimization technique, ed. IJEST. 2, Nueva York, Engg Journals Publication. Internationa Journal of Engineering Science and Technology, volumen 4, febrero de 2012: 564-570.

Pinto J. y Shayan E. Layout design of a furniture production line using formal methods, 1, Nueva York, JISE. Journal of Industrial and Systems Engineering, volumen 1, abril de 2007: pp. 81-96.

Riggs J. 2008. Sistemas de producción: planeación, análisis y control, México, Limusa Wiley, 2008, pp. 329-330.

Sanli H. y Eldemir F. Fatih University [en línea] 8 de diciembre de 2010 [Fecha de consulta: 14 de octubre de 2014]. Disponible en: www.mhi.org/downloads/learning/cicmhe/colloquium/.../eldemir.pdf.

Tompkins J. et al. Facilities planning, 2a ed., Neueva York, John Wiley \& Sons, Inc., 1996, pp. 382-384. 


\section{Este artículo se cita:}

Citación estilo Chicago

González, Longoria, Héctor Manuel. La heurística LDMTP: Una metodología híbrida basada en el problema de transporte para el diseño óptimo de la distribución de planta. Ingeniería Investigación y Tecnología, XVII, 04 (2016): 463-478.

\section{Citación estilo ISO 690}

González Longoria H.M. La heurística LDMTP: Una metodología híbrida basada en el problema de transporte para el diseño óptimo de la distribución de planta. Ingeniería Investigación y Tecnología, volumen XVII (número 4), octubre-diciembre 2016: 463-478.

\section{Semblanza del autor}

Héctor Manuel González-Longoria. Ingeniero químico por la Universidad Autónoma de Nuevo León. Maestro en educación superior por el Centro de Estudios Universitarios. Es subdirector de la carrera de procesos industriales área manufactura y responsable del cuerpo académico en la Universidad Tecnológica Santa Catarina, donde imparte las asignaturas de distribución de planta y cadena de suministros. Sus principales áreas de interés son las heurísticas aplicadas a temas de investigación de operaciones. 